Vanessa Tavares Pereira

Perfil de colonização de S.aureus em pacientes com dermatite atópica nas lesões agudas e crônicas

São Paulo 


\section{Vanessa Tavares Pereira}

\section{Perfil de colonização de S.aureus em pacientes com}

dermatite atópica nas lesões agudas e crônicas

Dissertação apresentada à Faculdade de Medicina da Universidade de São Paulo para obtenção do título de Mestre em Ciências

Programa de: Pediatria

Orientador: Dra. Ana Paula Beltran Moschione Castro

São Paulo 
Dados Internacionais de Catalogação na Publicação (CIP)

Preparada pela Biblioteca da

Faculdade de Medicina da Universidade de São Paulo

Creprodução autorizada pelo autor

Pereira, Vanessa Tavares

Perfil da colonização de S.aureus em pacientes com dermatite atópica nas lesões agudas e crônicas / Vanessa Tavares Pereira. -- São Paulo, 2017.

Dissertação(mestrado)--Faculdade de Medicina da Universidade de São Paulo.

Programa de Pediatria.

Orientadora: Ana Paula Beltran Moschione.

Descritores: 1.Dermatite atópica 2.Staphylococcus aureus 3.Qualidade de Vida 4.Criança 5.Resistência a meticilina 6.SCORAD

USP/FM/DBD-103/17 


\section{DEDICATÓRIA}

A Deus, pela oportunidade do burilamento terreno e todas as bênçãos concedidas ao longo desta jornada.

A minha mãe Graça pela dedicação absoluta, irrestrita e abdicação de sua vida em virtude da minha. Meu alicerce, minha vida, meu encontro de almas; sem você nada disso seria possível. Esta vitória é sua.

Ao meu pai Geraldo, meu ídolo e minha fonte de inspiração. Meu maior exemplo de professor brilhante e médico de homens e de almas.

Ao meu marido Thiago, companheiro nesta jornada por todo apoio e por acreditar, muitas vezes mais que eu, que seria possível. A minha eterna gratidão e amor.

Ao meu filho Rafael por embarcar comigo nesta aventura ainda intraútero e ser meu refúgio de amor e recuperação de energias para continuar buscando o melhor.

Ao meu irmão Fabio e meus sobrinhos Anthonio e Sophia pelo apoio e amor mesmo com tantas ausências.

A minha avó Dulce, meus tios e tias, primos e primas por toda acolhida e apoio sempre dispensado. 


\section{AGRADECIMENTOS}

A Dra. Ana Paula Moschione Castro, por aceitar este imenso desafio da orientação, conduzindo com amor, sabedoria e de forma brilhante cada passo desta jornada. Minha eterna gratidão.

Ao Dr. Antonio Carlos Pastorino, a prontidão em sempre contribuir com sua ampla experiência no que fosse preciso para o perfeito andamento da tese.

Ao Dr. Ulysses Doria Filho pela análise estatística

A Dra.Maria Fernanda Badue Pereira por toda colaboração e carinho

A Dra. Ana Lucia Guirau por todos os ensinamentos e incentivos de seguir adiante sempre.

A minha prima Márcia pela força nos momentos de desânimo e colo amigo sempre que preciso.

A minha amiga-irmã Luciana Dourado, pela acolhida e amizade ao longo destes anos.

A dona Marta pelo acolhimento e orações nos momentos de desanimo.

A Mariza pela atenção, carinho e revisão bibliográfica.

A Rosangela pela formatação da tese.

Ao Nivaldo, exemplo de amor e doação irrestrito, além do auxílio reprográfico desta dissertação. 
'Bré em li mesmo, age e verá os resultados.

Quando te esforcass a vida tambim se esforca frara to ajudar.

Bhico Savier 
Esta tese está de acordo com as seguintes normas, em vigor no momento desta publicação:

Referências: adaptado de International Committee of Medical Journals Editors (Vancouver)

Universidade de São Paulo. Faculdade de Medicina. Divisão de Biblioteca e Documentação. Guia de apresentação de dissertações, teses e monografias. Elaborado por Annelise Carneiro da Cunha, Maria Julia de A.L. Freddi, Maria F. Crestana, Marinalva de Souza Aragão, Suely Campos Cardoso, Valéria Vilhena. $3^{a}$ ed. São Paulo. Divisão de Biblioteca e Documentação: 2011.

Abreviaturas dos títulos dos periódicos de acordo com List of Journals Indexed in Index Medicus. 


\section{SUMÁRIO}

Lista de Abreviaturas e Siglas

Lista de Tabelas

Resumo

Abstract

1 INTRODUÇÃO............................................................................. 1

2 JUSTIFICATIVA............................................................................. 9

3 HIPÓTESE...................................................................................... 11

4 OBJETIVOS 13

5 MÉTODOS................................................................................... 15

6 RESULTADOS................................................................................. 21

6.1 Características Epidemiológicas, Clínicas e Hábitos de Higiene.... 22

6.2 Frequência e Perfil de Colonização do S.aureus......................... 23

6.3 Taxas de Colonização e Gravidade da Doença.......................... 26

7 DISCUSSÃO....................................................................................... 28

8 CONCLUSÕES......................................................................... 37

9 ANEXOS...................................................................................... 39

Anexo I Parecer do Comitê de Ética.............................................. 40

Anexo II Parceria com o Laboratório............................................... 41

Anexo III Critérios de Hanifin e Rajka.......................................... 42

Anexo IV Questionario de Perfil Clínico, Epidemiológico e Hábitos de Higiene................................................................... 43

10 REFERÊNCIAS....................................................................... 46 


\section{LISTAS DE ABREVIATURAS E SIGLAS}

\begin{tabular}{|c|c|}
\hline CAPPesq= & Comissão de Ética para Análise de Projetos de Pesquisa \\
\hline CLSI= & Clinicaland Laboratory Standards Institute \\
\hline $\mathrm{DA}=$ & Dermatite atópica \\
\hline HCFMUSP = & Hospital das Clínicas da Faculdade de Medicina \\
\hline & Universidade de São Paulo \\
\hline $\mathrm{ICr}=$ & Instituto da criança \\
\hline $\lg \mathrm{E}=$ & Imunoglobulina $E$ \\
\hline ISAAC = & International Study of Asthma and Allergies in Childhood \\
\hline $\mathrm{MHC}=$ & Complexo maior de histocompatibilidade \\
\hline $\mathrm{MRSA}=$ & Staphylococcus aureus meticilino - resistente \\
\hline S.aureus $=$ & Staphylococcus aureus \\
\hline $\mathrm{ORAD}=$ & Scoring atopic dermatitis \\
\hline
\end{tabular}




\section{LISTA DE TABELAS}

Tabela 1 Perfil epidemiológico dos pacientes com dermatite atópica. 22

Tabela 2 Perfil de colonização do S.aureus nos pacientes e cuidadores primários................................................... 23

Tabela 3 Perfil de sensibilidade dos S.aureus presentes nos sítios pesquisados de pacientes com DA e cuidadores primários................................................................. 24

Tabela 4 Avaliação da concordância do perfil de colonização do swab nasal do paciente $\mathrm{x}$ lesão aguda do paciente (kappa)................................................................... 24

Tabela 5 Avaliação da concordância do perfil de colonização do swab nasal do paciente $\mathrm{x}$ swab nasal do cuidador primário (kappa)

Tabela 6 Perfil de resistência antimicrobiana nos pacientes e cuidador primário

Tabela 7 Correlação dos níveis de prurido com 0 perfil de resistência do S.aureus em diversos locais de coleta 


\section{RESUMO}

Pereira VT.Perfil de colonização de S.aureus em pacientes com dermatite atópica nas lesões agudas e crônicas [Dissertação]. São Paulo: Faculdade de Medicina, Universidade de São Paulo, 2017.

INTRODUÇÃO: A dermatite atópica (DA) é considerada a principal e mais freqüente manifestação cutânea da atopia, podendo acometer até $20 \%$ da população pediátrica mundial. Trata-se de uma doença multifatorial onde agentes infecciosos como S.aureus são relevantes no desencadeamento das crises. OBJETIVOS: (1) Avaliar a taxa de colonização do S.aureus na pele e vestíbulo nasal de pacientes com DA e o seu perfil de sensibilidade a antibióticos (2) Comparar os resultados com a taxa de colonização do vestíbulo nasal dos cuidadores primários (3) Avaliar a associação entre colonização por S.aureus gravidade da doença e hábitos de higiene. MÉTODOS: Foi realizado estudo prospectivo entre dezembro de 2016 a fevereiro de 2017. Foram avaliados aspectos clínicos, epidemiológicos e laboratoriais de crianças com DA em seguimento na unidade de alergia e imunologia do Instituto da Criança da Faculdade de Medicina da Universidade de São Paulo (ICr HCFMUSP). Foram incluídos pacientes que apresentaram DA moderada ou grave, classificados de acordo com o escore de gravidade SCORAD (scoring atopic dermatitis). Foram excluídos pacientes que haviam utilizado antibioticoterapia há menos de 30 dias da inclusão, que apresentavam infecções cutâneas ou utilizavam imunossupressores por via sistêmica. A pesquisa de S.aureus em pacientes foi realizada por meio de coleta de swab em vestíbulo nasal, lesão aguda e crônica dos pacientes com DA e nos cuidadores primários foi realizado swab de vestibulo nasal. RESULTADOS: Foram avaliados 27 pacientes (14M), 18 com DA moderada e 9, grave. O início da doença ocorreu entre 2 e 60 meses de idade (mediana de 12m) e a coleta de swab ocorreu ao 9 anos (mediana, com variação entre 2 e 15 anos). Todos os pacientes apresentaram colonização por S.aureus em pelo menos um local de coleta, sendo a lesão aguda o mais frequente (24/27), seguido do nariz (23/27) e lesão crónica (20/27). Dezenove cuidadores apresentaram S.aureus em vestíbulo nasal. A presença de S.aureus meticilino resistente (MRSA) foi detectada em 9/23 pacientes no vestíbulo nasal, 8/24 na lesão aguda e 6/20 na lesão crônica. Entre os cuidadores 6/19 apresentaram S.aureus MRSA em vestíbulo nasal. Pacientes e cuidadores primários apresentaram semelhança no perfil de sensibilidade a antibióticos (kappa 0,75). A gravidade da doença (SCORAD), não se correlacionou de maneira significante com a taxa de colonização, mas pacientes com escores de prurido mais elevados apresentaram estafilococos MRSA com maior freqüência de maneira estatisticamente significante $(p=0,29)$. Houve uma elevada taxa de compartilhamento de produtos com destaque: produtos pessoais (17/27) cama (11/27) e toalha (3/27) CONCLUSÂO: S.aureus permanece como uma bactéria bastante presente na pele de quem apresenta dermatite atópica, independentemente do tipo de lesão. A 
presença de elevadas taxas de S.aureus MRSA é um sinal de alerta para o cuidadoso manejo de pacientes com DA e estratégias que envolvam a orientação com relação as medidas de higiene e cuidado pessoal devem fazer parte do tratamento da dermatite atópica.

Descritores: Dermatite atopica, Staphylococcus aureus, qualidade de vida, criança, resistência a meticilina, scorad 
Pereira VT. Profile of S.aureus colonization in patients with atopic dermatitis in acute and chronic lesion [Dissertation]. São Paulo: "Faculdade de Medicina, Universidade de São Paulo"; 2017.

\section{ABSTRACT}

Atopic dermatitis (AD) is considered the main and most common cutaneous manifestation of atopy, affecting up to $20 \%$ of the world pediatric population. It is a multifactorial disease where infectious agents such as $S$. aureus are relevant in the onset of crises. OBJECTIVES: (1) To evaluate the rate of colonization of S.aureus in the skin and nasal vestibule of patients with $A D$ and its antibiotic susceptibility profile. (2) To compare the results with the nasal vestibular colonization rate of primary caregivers (3) Evaluate the association between S.aureus colonization, disease severity and hygiene habits. METHODS: A prospective study was carried out between December 2016 and February 2017. Clinical, epidemiological and laboratory aspects of children with $A D$ were evaluated at the unit of allergy and immunology of the Children's Institute of the School of Medicine of the University of São Paulo (ICr HCFMUSP). Patients with moderate or severe AD, classified according to SCORAD (scoring atopic dermatitis) severity score were included. Patients who used antibiotic therapy less than 30 days before inclusion, who had cutaneous infections or who used systemic immunosuppressants were excluded. The study of S.aureus in patients was performed through nasal vestibule swab collection, acute and chronic lesion of patients with $A D$, and nasal vestibule swabs were performed in primary caregivers. RESULTS: Twenty-seven patients (14M), 18 patients with moderate $A D$ and 9 patients, were diagnosed. The onset of the disease occurred between 2 and 60 months of age (median of $12 \mathrm{~m}$ ) and swab collection occurred at 9 years (median, Between 2 and 15 years). All patients presented colonization by S.aureus in at least one collection site, with acute lesion being the most frequent (24/27), followed by the nose (23/27) and chronic lesion (20/27). Nineteen caregivers presented $S$.aureus in the nasal vestibule. The presence of methicillin-resistant S.aureus (MRSA) was detected in 9/23 patients in the nasal vestibule, $8 / 24$ in the acute lesion and 6/20 in the chronic lesion. Carriers 6/19 presented S.aureus MRSA in the nasal vestibule. Patients and primary caregivers presented similarity in the sensitivity profile to antibiotics (kappa 0.75). The severity of the disease (SCORAD) did not correlate significantly with the rate of colonization, but patients with higher pruritus scores had statistically more significant MRSA staphylococci $(p=0.29)$. There was a high rate of product sharing with emphasis on: personal products (17/27) bed (11/27) and towel (3/27) CONCLUSION: S.aureus remains a very present bacterium in the skin of those with atopic dermatitis, Regardless of the type of injury. The presence of high rates of S.aureus MRSA are a warning sign for the careful management of patients with $A D$ and strategies that involve guidance 
regarding hygiene measures and personal care should be part of the treatment of atopic dermatitis.

KEYWORDS: Dermatitis,atopic; Staphylococcus aureus; Child, Quality of life, Methicillin-Resistence, scorad 
1. INTRODUÇÃO 
A dermatite atópica (DA) ou eczema atópico é considerada a principal e mais frequente manifestação cutânea da atopia, podendo acometer até $20 \%$ da população pediátrica mundial ${ }^{1}$. No Brasil, graças aos dados do estudo epidemiológico ISAAC (International Study of Asthma and Allergies in Childhood), calcula-se 8,2\% das crianças entre 6 e 7 anos (com variações entre 5,3 e 15\%) apresentem DA e entre os adolescentes de 13 e 14 anos, 10 a 14\% referiram o diagnóstico da doença ${ }^{2}$. Tem-se observado um aumento da prevalência da doença na Europa, entretanto no Brasil, somente as formas mais graves da doença é que apresentaram elevação entre 0,5 a $1 \%{ }^{2}$. Este crescimento das formas mais graves segundo o mesmo estudo foi atribuído a mudanças no estilo de vida e padrão alimentar, com destaque a menor ingestão de ácidos graxos essenciais e alimentos ricos em fibras ${ }^{2}$. $\mathrm{O}$ contato mais precoce com substâncias irritantes, a presença de alérgenos em produtos de higiene pessoal e a maior exposição à alérgenos ambientais também foram descritos como possíveis fatores responsáveis pelo aumento da prevalência de $\mathrm{DA}^{1,3,4}$

A DA é considerada uma doença resultante da interação entre componentes genéticos, fatores ambientais, defeitos da barreira cutânea e fatores imunológicos. Portanto, trata-se de uma doença genética, de herança poligênica, com evidentes alterações imunológicas, fortemente influenciadas por fatores ambientais que comprometem a qualidade de vida do paciente. Muitas vezes há um grande prejuízo do sono, agravado pelo prurido intenso, de difícil manejo levando a frustração do paciente e sensação de impotência do cuidador diante destes pacientes ${ }^{5}$. Estima-se que pais de crianças com 
dermatite atópica percam cerca de 2,5 horas de sono por noite e irmãos podem apresentar distúrbios comportamentais. Por fim, trata-se de uma doença de caráter crônico com comprometimento importante da qualidade de vida das crianças, mas também de familiares ${ }^{6}$.

A população pediátrica é especialmente comprometida, cerca de $85 \%$ dos casos novos se iniciam até os 5 anos de idade sendo $50 \%$ deles até o final do primeiro ano de vida ${ }^{7}$. Na etiopatogenia da DA, múltiplos fatores parecem fazer parte de um complexo mecanismo que resulta em intensa inflamação e comprometimento da barreira cutânea, há uma ativação do sistema imunológico, em especial dos linfócitos T auxiliares, eosinófilos e grande produção de $\lg ^{8}$. Pacientes com dermatite atópica podem apresentar: maior concentração de $\mathrm{Fc \varepsilon RI}$ (receptor de alta afinidade para IgE) nas células dendríticas, menor síntese de peptídeos antimicrobianos, macrófagos com síntese aumentada de determinadas citocinas e basófilos com maior facilidade de degranulação ${ }^{9}$. Setores da imunidade adquirida também estão comprometidos: pacientes apresentam maior síntese de lgE, maior possibilidade de síntese de lgE específica para alérgenos diversos, disfunção de linfócitos TCD8, o que pode facilitar mais infecções cutâneas especialmente por vírus e, finalmente, maior tendência à produção de linfócitos TCD4 com perfil de citocinas do tipo Th2, que facilita o infiltrado de eosinófilos e síntese de $\operatorname{lgE}^{8,9}$. Cerca de $50 \%$ dos pacientes com DA apresentam mutações nos genes das filagrinas, proteínas que compõe o cimento intercelular garantindo a integridade do estrato córneo, como consequência há aumento da perda transepidermica de água, ressecamento 
da pele e intensificação do prurido. Nestes pacientes há ainda maiores chances de desenvolvimento de outras doenças alérgicas como rinite alérgica, asma e alergia alimentar ${ }^{6,10}$.

Uma série de fatores do ambiente pode contribuir para o desencadeamento ou agravamento das lesões cutâneas com destaque: irritantes diretos na pele, alérgenos alimentares, aeroalérgenos e agentes infecciosos ${ }^{9}$. Alguns destes agentes desencadeantes podem atuar como fatores epigenéticos facilitando a expressão gênica e ampliando a inflamação ${ }^{11}$.

Entre os agentes infecciosos, é importante destacar o Staphylococcus aureus (S.aureus) como um dos mais importantes desencadeadores de crises, uma vez que sua interação com a pele de pacientes com DA pode facilitar a ativação da doença ${ }^{12}$. Um estudo realizado no Brasil, por Petry e colaboradores em 2013, documentou a elevada presença de S.aureus na pele dos pacientes com DA, entre 76 a $100 \%$ de culturas positivas naqueles que apresentam pele lesionada e 30 a $100 \%$ dos indivíduos com pele íntegra, sendo bastante superior aos 5 a 30\% observados em indivíduos saudáveis ${ }^{12,13}$. Esta suscetibilidade da pele do paciente à colonização pelo estafilococo está relacionada a fatores diversos. As alterações na barreira cutânea, com destaque à perda de integridade, em decorrência de um desequilíbrio estrutural da matriz lipídica extracelular, permitem a ação de irritantes e reduzem o limiar do prurido, facilitando o trauma da coçadura e o consequente prejuízo da arquitetura da pele. $O$ ato de coçar induz a colonização bacteriana por acarretar solução de continuidade da barreira 
cutânea do paciente com DA e expor os ligantes laminina e fibronectina. Uma vez expostos, estes ligantes fixam adesinas, que estão localizados na parede celular do S.aureus, permitindo sua maior ancoragem ${ }^{11}$.

Outro mecanismo facilitador da permanência desta bactéria na pele é o desbalanço lipídico que ocorre na derme destes pacientes. As ceramidas são as principais moléculas de retenção de água no espaço extracelular do envelope cornificado. Estas ceramidas, em especial as sub frações 1 e 3, estão significativamente reduzidas na $D A$, tanto na pele sã como nas lesões ${ }^{14}$. A composição alterada dos lipídeos do estrato córneo leva ao aumento da permeabilidade cutânea e facilita a penetração e permanência das bactérias. O S.aureus por sua vez, produz enterotoxinas (enterotoxinaestafilocócica A-SEA; enterotoxinaestafilocócica B-SEB; toxina da síndrome do choque tóxico-TSST-1) que podem atuar como superantígenos, estimulando de forma policlonal linfócitos T e macrófagos sem a interferência do sistema MHC (complexo maior de histocompatibilidade), além de liberar citocinas pró-inflamatórias, que estimulam o sistema imunológico cutâneo, exacerbando a DA. A desregulação imunológica presente nestes pacientes facilita a gênese de anticorpos $\lg$ E específicos para as exotoxinas, ou seja, os superantígenos podem atuar como alérgenos, uma vez que $57 \%$ dos doentes com DA apresentam $\lg$ E contra as enterotoxinas ${ }^{11,15}$. Luger e colaboradores mostraram ainda que existe correlação da gravidade da DA e a presença de anticorpos específicos contra os superantígenos ${ }^{16,17}$. A correlação entre a gravidade da DA e a colonização por S.aureus, tem demonstrado ser um 
importante mecanismo de agravamento das lesões de pele e, neste contexto, é importante estar atento a mudanças no perfil de sensibilidade a antibióticos desta bactéria ${ }^{15,18}$.

Durante os últimos 10 anos, tem sido observado o desenvolvimento de resistência a agentes antimicrobianos comumente prescritos ${ }^{18}$. A infecção por Staphylococcus aureus meticilino-resistente (MRSA), surgiu a primeira vez nos Estados Unidos em 1970 e em 1990 foi considerada endêmica na maioria dos grandes centros médicos urbanos. MRSA se tornou endêmico em hospitais no mundo todo e agora é considerado patógeno amplamente existente em diversas regiões geográficas ${ }^{13,14,19}$.

Tradicionalmente as infecções causadas por MRSA eram limitadas aos hospitais, porém recentemente têm sido associadas a processos infecciosos adquiridos na comunidade (CA-MRSA $)^{18}$. A infecção por estas bactérias podem contribuir para a exacerbação da $\mathrm{DA}^{19}$. Farajzadeh et al. 2008, analisaram o swab de 50 pacientes com dermatite atópica no Irã e identificaram cultura positiva em $74 \%$ destes pacientes ${ }^{20}$. Em outro estudo 0 S.aureus foi isolado em $66 \%$ dos casos, onde $90 \%$ eram resistentes à penicilina, $66.7 \%$ a eritromicina e $13.8 \%$ eram resistentes a cefalexina ${ }^{17}$. Estes achados corroboram com a elevada capacidade que o estafilococo tem de desenvolver resistência, fenômeno que também tem sido observado nos pacientes com dermatite atópica ${ }^{17}$.

A dermatite atópica, por seu caráter crônico, pode apresentar oscilações em suas manifestações clínicas variando entre lesões agudas e crônicas. Nos períodos de agudização da doença, há piora do prurido e das 
lesões que se tornam mais exsudativas e hiperemiadas. Em algumas lesões agudas há sinais evidentes de infecção, mas em outras pode-se supor a presença da bactéria como facilitador de inflamação e, em alguns casos, a antibioticoterapia é proposta de maneira empírica na tentativa de minimizar os sinais e sintomas. Entretanto esta intervenção terapêutica pode ser uma das causas do aumento dos casos de infecções por estafilococos MRSA ${ }^{13}$.

A elevada prevalência de DA no Brasil demanda a necessidade de avaliar o percentual de colonização destes pacientes e o perfil de resistência desta bactéria. Há no Brasil, uma escassez de estudos avaliando a colonização por S.aureus em pacientes com dermatite atópica. No Rio Grande do Sul um grupo de pesquisadores avaliou 93 pacientes com DA, nos quais $27 \%$ apresentaram colonização por S.aureus nas narinas e $60 \%$ na lesão aguda. Neste estudo não foram coletados swab de lesão crônica ${ }^{13}$. No Rio de Janeiro, estudo realizado no Hospital Universitário Federal do Rio de Janeiro, mostrou que $85 \%$ pacientes apresentaram colonização por S.aureus em narinas e, destes, 86,6\%, apresentaram valores de SCORAD, um escore de gravidade para dermatite atópica, moderados e 13,4\% pacientes apresentaram valores de SCORAD grave ${ }^{21}$.

A avaliação de hábitos de higiene e condições de moradia de pacientes com dermatite atópica pode contribuir para o melhor entendimento do comportamento da DA em especial do perfil de infecções. Estudo realizado entre 2012 e 2013 por Fritz na universidade de Washington, avaliou os hábitos de higiene de 50 crianças colonizadas por S.aureus e pesquisou a presença de S.aureus nas superfícies de suas residências. Pode-se concluir, 
com base neste estudo, que o S.aureus consegue sobreviver em objetos inanimados por períodos prolongados e que um maior número de indivíduos por $\mathrm{m}^{2}$ também está associado à maior proporção de superfícies contaminadas com S.aureus, facilitando maior exposição à bactéria e possível exacerbação da $D A^{22}$. Portanto é importante avaliar estes hábitos de higiene nos pacientes de DA no Brasil. Outro aspecto interessante e ainda pouco abordado é a avaliação do perfil de colonização comparando-se diversos sítios do mesmo paciente ${ }^{13,21}$. 
Em virtude deste aumento das infecções assim como aumento do potencial de virulência e maior prevalência na comunidade, considerando a necessidade de ampliar o conhecimento do perfil de colonização e infecção pelo S.aureus, elaborou-se o presente estudo. A unidade de alergia e imunologia do instituto da criança segue pacientes com dermatite atópica moderada ou grave há cerca de 15 anos e no momento conta com mais de uma centena de pacientes em seguimento regular. Durante todo este período pode-se observar a presença de um grupo de pacientes com quadros evidentes de infecção e em outro grupo apenas a intensa hiperemia demandou a utilização de antibioticoterapia. Faz-se necessária a documentação e o entendimento da necessidade real da utilização de antibioticoterapia para que se possam minimizar os riscos de resistência bacteriana, mas também cuidar adequadamente do paciente a fim de se elevar a autoestima e o aumento da qualidade de vida das nossas crianças. Acrescentar a esta análise um entendimento sobre condições de higiene e hábitos de vida pode contribuir para melhor avaliação do paciente e possíveis intervenções futuras. 
Pacientes com dermatite atópica apresentam uma maior frequência de colonização por S.aureus nas lesões agudas em relação à população sem dermatite atópica. 


\section{OBJETIVOS}




\section{Geral}

- Descrever a frequência de colonização e a sensibilidade aos antibióticos do S.aureus na pele dos pacientes com dermatite atópica nas lesões agudas, crônicas e vestíbulos nasais e comparar com a frequência de colonização do vestíbulo nasal do seu cuidador primário.

\section{Específico}

- Avaliar a associação entre colonização por S.aureus em pacientes com DA, a gravidade da doença e hábitos de higiene. 
O presente estudo foi aprovado no Comitê de ética e pesquisa de acordo com o parecer 1.637 .841 (anexo I) e realizado em parceria com o laboratório de microbiologia do HC/FMUSP (anexo II)

Foram estudados 27 pacientes em acompanhamento na unidade de alergia e imunologia, do ambulatório de dermatite atópica do ICRHC/FMUSP. Este tamanho amostral conferiu ao estudo $80 \%$ de poder (variação entre 53 e 83\%) para encontrar uma frequência de colonização pelo S.aureus (GraphPad Stat Mate). A seleção dos pacientes foi feita pela ordem de chegada e realizada no período de dezembro de 2016 a fevereiro de 2017. Para participarem desta pesquisa os pacientes respeitaram todos os critérios de inclusão abaixo apresentados:

\section{Critérios de Inclusão}

1. Diagnóstico confirmado de dermatite atópica segundo os critérios de Hanifin e Rajka, $1980^{23}$, o qual considera dermatite atópica confirmada a presença de 3 critérios clínicos maiores e 3 ou mais critérios clínicos menores (anexo III).

2. Presença de dermatite atópica moderada ou grave de acordo com escore de gravidade SCORAD (Scoring atopic dermatitis) que mede a gravidade e a extensão da DA, sendo considerados leves pacientes com pontuação entre 0 -15, DA moderada $15-50$ e DA grave $>50^{24}$.

3. Presença de lesão aguda definida por hiperemia, exsudato e acentuação do prurido sem sinais de infecção associada ${ }^{25}$. 
4. Presença concomitante de lesão crônica definida por presença de liquenificação (Nanette et al, 2016) ${ }^{25}$.

5. Possuir um cuidador primário que permitisse a coleta de swab nasal para identificação de S.aureus, sem a utilização previa de antibioticoterapia nas últimas 4 semanas e ausência de processo infeccioso bacteriano. Cuidador primário foi definido como a pessoa que efetivamente cuida do paciente com dermatite atópica e que more na mesma residência.

\section{Critérios de exclusão}

Foram excluídos os pacientes que apresentaram:

1. Lesões características de impetigo, furúnculos ou celulite.

2. Uso de antibióticos há menos de um mês da coleta do material.

3. Uso de imunossupressores sistêmicos

Após respeitados aos critérios de inclusão e exclusão respectivamente, os responsáveis dos candidatos selecionados foram convidados a participar da pesquisa, assinando os termos de consentimento livre e esclarecido e assentimento, quando pertinente.

Todo o processo de investigação e coleta do material foi feito em uma única visita médica. Os pacientes foram caracterizados de acordo com os aspectos epidemiológicos, clínicos, perfil sócio econômico e hábitos de higiene. 
O perfil clínico e epidemiológico dos pacientes com Dermatite Atópica incluiu dados pertinentes à pesquisa e foi obtido através de um questionário padronizado para o atendimento na unidade (anexo IV).

A caracterização dos hábitos de higiene foi feita através de um questionário que contemplou aspectos relacionados a cuidados diários e hábitos familiares, baseados nos resultados encontrados por FRITZ e colaboradores, $2014^{22}$ (anexo IV).

A pesquisa de S.aureus em pacientes com dermatite atópica e cuidadores primários foi realizada por meio de coleta de swab de fossas nasais anteriores, swab de lesão aguda e swab de lesão crônica dos pacientes com DA. Nos cuidadores primários, o sitio de coleta foi a fossa nasal. O próprio swab foi utilizado para coleta e transporte de amostras imersas em meio STUART da marca Absorve ${ }^{\circledR}$. O procedimento de coleta foi indolor e simples, sendo realizado pela pesquisadora. A técnica de coleta em vestíbulo nasal envolveu: inspeção do nariz, deslocando-se a ponta do nariz para cima com o dedo polegar e inclinando-se a cabeça do sujeito da pesquisa. Foi introduzido o swab na cavidade nasal direcionando-o para cima (direção dos olhos), com uma angulação de 30 a 45 graus em relação seu lábio superior. Depois foi removido o coletor das fossas nasais cuidadosamente e colocado imediatamente no tubo com solução fisiológica. Para a coleta de material nas lesões agudas e crônicas, deslizou-se o swab suavemente colocando-o em seguida no meio de conservação. As amostras coletadas foram levadas em seguida ao laboratório central de microbiologia HC/FMUSP. 
O cultivo das amostras foi feito em ágar sangue, em conformidade com a rotina do laboratório de microbiologia. A identificação das cepas de $S$. aureus foi feita usando a metodologia de Espectrometria de massa (MaldiTof) (Koubek et al, 2012; Goulart et al, 2013) ${ }^{26,27}$. A avaliação de susceptibilidade aos antimicrobianos recomendados pelo Clinicaland Laboratory Standards Institute (CLSI) foi realizada usando microdiluição automatizada (VITEK 2KL) (Jorgesen et al, 2009) ${ }^{28}$. As cepas de S.aureus isoladas e identificadas foram guardadas em Banco de Cepas do laboratório de microbiologia e congeladas para posterior analise genética.

\section{Análise estatística}

Os dados relacionados às características epidemiológicas e clínicas foram apresentados de maneira descritiva em tabelas para caracterização da amostra avaliada.

Para análise da significância foram utilizados os testes Qui-Quadrado nos três locais pesquisados no paciente (vestíbulo nasal, lesão aguda e lesão crônica). Para avaliação de concordância entre o perfil de colonização do swab nasal de pacientes e cuidadores primários foi utilizado o teste kappa. Serão consideradas coincidentes kappa de pelo menos 0,70 para indicar coincidência.

Os dados nominais (sexo, antecedentes pessoais e familiares de atopia, hábitos de higiene) foram descritos através suas freqüências. A concordância entre positividade da cultura no paciente e no cuidador 
primário foi investigada através teste Kappa. As variáveis contínuas (idade na coleta e no início dos sintomas, escore de gravidade) foram descritas através de medianas, valores máximos e mínimos. As medianas dos escores de gravidade e níveis de prurido nos grupos colonizados e não colonizados foram comparadas através teste de Mann-Whitney.

Em todas as análises foi adotado um nível de significância de 5\%, tendo sido utilizado o software SPSS 13.0 para realizá-las. 


\subsection{Características Epidemiológicas, Clínicas e Hábitos de Higiene}

Entre os 27 pacientes avaliados, $N=27(51,8 \%)$ eram do sexo masculino e apresentaram mediana de idade na data da coleta de 09 anos (Variação 02 a 15 anos). A mediana de idade de inicio dos sintomas foi de 12 meses (variação de 02 a 60 meses) e a maioria dos pacientes 18 (66,6\%) apresentou dermatite atópica moderada. Vinte e três (85\%) pacientes incluídos apresentaram outras doenças alérgicas com destaque a rinite e asma e $20(74 \%)$ do total apresentaram antecedentes familiares de atopia. Com relação aos hábitos de higiene $62,9 \%$ dos pacientes compartilhavam produtos pessoais como; escova de dente, escova de cabelo, cortadores de unhas, talheres, toalhas de banho e cama. (Tabela 1).

Tabela 1 - Perfil epidemiológico dos pacientes com dermatite atópica

\begin{tabular}{lc}
\hline Variáveis & Pacientes (N=27) \\
\hline \hline Sexo & $14(51,8 \%)$ \\
Masculino & \\
Idade & $9(2-15)$ \\
Idade na coleta (ano) & $12(2-60)$ \\
Idade de início dos sintomas (meses) & \\
Gravidade (SCORAD) & $18(66,6 \%)$ \\
Moderado & $9(33,3 \%)$ \\
Grave & \\
Antecedentes pessoais de atopia & $23(85 \%)$ \\
Sim & \\
Antecedentes familiares de atopia & $20(74 \%)$ \\
Sim & \\
Hábitos de higiene & $1(3,7 \%)$ \\
Compartilha escova de dente & $3(11,1 \%)$ \\
Compartilha toalha & $11(40,7 \%)$ \\
Compartilha cama & $17(62,9 \%)$ \\
Compartilha produtos pessoais & $20(74 \%)$ \\
Lava as mãos antes das refeições &
\end{tabular}




\subsection{Frequência e Perfil de Colonização do S.aureus}

Vinte e quatro pacientes apresentaram positividade para o S.aureus em pelo menos uma amostra coletada e em 19 cuidadores primários também foi detectada a presença S.aureus em vestíbulo nasal (Tabela 2).

Tabela 2- Perfil de colonização do S.aureus nos pacientes e cuidadores primários

\begin{tabular}{ccc}
\hline SWAB & Pacientes $(\mathbf{N}=27)$ & Cuidador Primário (N=27) \\
\hline \hline Nasal paciente & $23(85 \%)$ & \\
Lesão aguda & $24(88 \%)$ & \\
Lesão crônica & $20(74 \%)$ & $19(70 \%)$ \\
Nasal cuidador primário & & 190 \\
\hline
\end{tabular}

Entre os pacientes colonizados por S.aureus houve um predomínio de cepas de perfil sensível a meticilina, assim como entre os cuidadores primários colonizados (Tabela 3). Entretanto, em todos os locais de pesquisa de colonização, pelo menos um terço das cepas isoladas apresentou resistência à meticilina.

Não houve diferença estatisticamente significante entre o perfil de colonização do paciente comparado com o seu cuidador primário, nem com relação ao perfil de sensibilidade $(p>0,05)$. Assim como não houve diferença entre o percentual de colonização nasal e da lesão aguda dos pacientes com DA. 
Tabela 3 - Perfil de sensibilidade dos S.aureus presentes nos sítios pesquisados de pacientes com DA e cuidadores primários

\begin{tabular}{rc}
\hline \multicolumn{1}{c}{ Perfil de sensibilidade } & N 27 (\%) \\
\hline \hline Agente Nasal Paciente (N= 23) & $14(60)$ \\
MSSA & $9(39)$ \\
Agente Lesão Aguda (N= 24) & \\
MSSA & $16(66)$ \\
MRSA & $8(33)$ \\
Agente Lesão Crônica (N= 20) & \\
MSSA & $14(70)$ \\
Agente Nasal Cuidador primário (N= 19) & $6(30)$ \\
MSSA & $13(68)$ \\
MRSA & $6(31,5)$ \\
\hline
\end{tabular}

Houve concordância entre o perfil de sensibilidade do S.aureus em swab de vestíbulo nasal dos pacientes com os resultados obtidos nas lesões agudas (Tabela 4).

Tabela 4 - Avaliação da concordância do perfil de colonização do swab nasal do paciente $\mathrm{x}$ lesão aguda do paciente (kappa)

\begin{tabular}{ccc}
\hline \multirow{2}{*}{ VESTIBULO NASAL PACIENTE } & \multicolumn{2}{c}{ LESÃO AGUDA PACIENTE } \\
\cline { 2 - 3 } & MSSA & MRSA \\
\hline \hline MSSA & 11 & 1 \\
MRSA & 1 & 7 \\
\hline Total & $12(76.9 \%)$ & $8(23.1 \%)$ \\
\hline
\end{tabular}

Kappa das categorias: 0,792, geral: 0,792 $p<0,001$ 
Pacientes e cuidadores primário compartilharam o mesmo perfil de resistência. A análise da concordância mostrou significância estatística entre os S.aureus presentes no vestíbulo nasal do paciente e de seu cuidador primário com predomínio de cepas sensíveis a meticilina.

Tabela 5 - Avaliação da concordância do perfil de colonização do swab nasal do paciente x swab nasal do cuidador primário (kappa)

\begin{tabular}{ccc}
\hline \multirow{2}{*}{ AGENTE NASAL PACIENTE } & \multicolumn{2}{c}{ AGENTE NASAL CONTACTANTE } \\
\cline { 2 - 3 } & MSSA & MRSA \\
\hline \hline MSSA & 8 & 0 \\
MRSA & 1 & 7 \\
\hline Total & 9 & 7 \\
\hline Kappa das categorias: 0,875, geral: $0,875 p<0,001$ &
\end{tabular}

Considerando o perfil de resistência aos antibióticos testados: gentamicina, linezolida, moxifloxacino, rifampicina, sulfa + trimetropin, teicoplamina, tigenicilina e vancomicina, observou-se sensibilidade em $100 \%$ das amostras coletadas. (Tabela 6). 
Tabela 6 - Perfil de resistência antimicrobiana nos pacientes e cuidador primário

\begin{tabular}{|c|c|c|c|c|}
\hline ANTIBIOGRAMA & $(\mathrm{N}=23)$ & $\begin{array}{l}\text { Lesão } \\
\text { aguda } \\
(\mathrm{N}=24)\end{array}$ & $\begin{array}{c}\text { Lesão } \\
\text { crônica } \\
(\mathrm{N}=20)\end{array}$ & $\begin{array}{c}\text { Cuidador primário } \\
\qquad(\mathrm{N}=19)\end{array}$ \\
\hline Penicilina & 17 (85\%) & $13(59 \%)$ & $12(63 \%)$ & 15 (94\%) \\
\hline Eritromicina & $11(55 \%)$ & $9(41 \%)$ & $6(32 \%)$ & 7 (44\%) \\
\hline Oxacilina & $9(39 \%)$ & $8(33 \%)$ & $6(30 \%)$ & $6(31,5 \%)$ \\
\hline Clindamicina & $5(25 \%)$ & $4(18 \%)$ & $3(16 \%)$ & $4(25 \%)$ \\
\hline Ciprofloxacino & $0(0 \%)$ & $0(0 \%)$ & $0(0 \%)$ & $0(0 \%)$ \\
\hline Gentamicina & $0(0 \%)$ & $0(0 \%)$ & $0(0 \%)$ & $0(0 \%)$ \\
\hline Linezolida & $0(0 \%)$ & $0(0 \%)$ & $0(0 \%)$ & $0(0 \%)$ \\
\hline Moxifloxacino & $0(0 \%)$ & $0(0 \%)$ & $0(0 \%)$ & $0(0 \%)$ \\
\hline Rifampicina & $0(0 \%)$ & $0(0 \%)$ & $0(0 \%)$ & $0(0 \%)$ \\
\hline Sulfa + Trimetropin & $0(0 \%)$ & $0(0 \%)$ & $0(0 \%)$ & $0(0 \%)$ \\
\hline Teicoplamina & $0(0 \%)$ & $0(0 \%)$ & $0(0 \%)$ & $0(0 \%)$ \\
\hline Tigenicilina & $0(0 \%)$ & $0(0 \%)$ & $0(0 \%)$ & $0(0 \%)$ \\
\hline Vancomicina & $0(0 \%)$ & $0(0 \%)$ & $0(0 \%)$ & $0(0 \%)$ \\
\hline
\end{tabular}

\subsection{Taxas de Colonização e Gravidade da Doença}

A média do valor do SCORAD, escore de gravidade, não variou entre os pacientes colonizados e os não colonizados ( $p>0,05$, Teste de Mann Whitney). Também não foi observada variação estatisticamente significante entre gravidade da doença e colonização por S.aureus meticilino sensíveis ou S.aureus meticilino resistentes ( $p>0,05$, Teste de Mann Whitney). Maiores níveis de prurido foram relacionados de maneira significante a maiores taxas S.aureus meticilino resistente (MRSA) em vestíbulo nasal (Mann Whitney) (Tabela 7). 
Tabela 7- Correlação dos níveis de prurido com o perfil de resistência do S.aureus em diversos locais de coleta

\begin{tabular}{ccc}
\hline S. aureus (local de coleta) & $\begin{array}{c}\text { Escore de prurido e } \\
\text { variação }\end{array}$ & P \\
\hline \hline Vestíbulo Nasal & $5(0-10)$ & 0,029 \\
MSSA $(n=14)$ & $8(5-10)$ & \\
MRSA $(n=9)$ & & 0,0976 \\
Lesão Aguda (N=24) & $5(0-10)$ & \\
MSSA $(n=16)$ & $8(2-10)$ & \\
MRSA $(n=8)$ & $10(76.9)$ & \\
Lesão Crônica (N=20) & $3(23.1)$ & \\
MSSA $(n=14)$ &
\end{tabular}

Não houve associação significante entre hábitos de higiene e gravidade da doença $(p>0,05)$. 
A dermatite atópica é uma doença que apresenta fisiopatologia complexa e envolve aspectos genéticos e ambientais que culminam com um processo inflamatório intenso e comprometimento da barreira cutânea ${ }^{7}$. Neste contexto, o S.aureus exerce papel relevante no desencadeamento das lesões e piora do quadro dermatológico ${ }^{7,8}$. Um conjunto de fatores contribui para isto com destaque a fragilidade na barreira cutânea associado a facilidades de ancoragem do S.aureus ${ }^{9}$. BOGUNIEWICZ et al, 2010, nesta revisão, justificaram a susceptibilidade aumentada a infecções e colonização cutânea nestes pacientes pela presença da expressão de vários genes que acabam por contribuir para a desregulação imunológica ${ }^{33}$. Um exemplo desta disfunção é a possível inibição dos mecanismos de destruição do S.aureus pelas ações da IL4 e IL13, aumentadas na DA ${ }^{34}$. Além disto, observou-se as anormalidades na imunidade inata, incluindo redução de peptídeos antimicrobianos, diminuição do recrutamento de células, como neutrófilos para a pele e defeitos dos receptores toll-like ${ }^{35}$, assim como anormalidades da barreira epidérmica como já mencionado anteriormente.

As evidencias clinicas da participação desta bactéria na ativação da DA estão relacionadas à melhora dos pacientes quando se utiliza a antibioticoterapia. Esta prática, no entanto, pode resultar no aumento da resistência bacteriana e na dificuldade do manejo da $\mathrm{DA}^{12}$.

O presente estudo pode documentar a relevância do S.aureus na pele de pacientes com dermatite atópica. Os elevados dados de colonização encontrados, até $88 \%$ de presença desta bactéria dependendo do local 
pesquisado, reiteram a importância desta documentação. Estudos prévios já haviam descrito esta intensa associação do S.aureus nos pacientes com dermatite atópica, através da coleta de swab nasal e / ou da pele dos pacientes com dermatite atópica. Gomes et al 2010, por exemplo, coletaram swab nasal e de pele de 100 pacientes com dermatite atópica moderada a grave em hospital universitário do Siri Lanka, onde 57\% apresentaram colonização por S.aureus na pele. No Brasil em 2013, LIPNHARSKI et al realizaram análise semelhante, porém agora coletando swab da pele do paciente com DA e dos indivíduos saudáveis e identificaram que 5 a $30 \%$ destes indivíduos apresentavam a pele colonizada por S.aureus, enquanto os pacientes com DA 76 a $100 \%$ estavam colonizados $^{12}$. Em 2015, no Rio de Janeiro, Cavalcante et al, encontraram prevalência semelhante a este estudo com $85 \%$ de colonização do swab nasal nos pacientes com DA.

Outra contribuição deste estudo foi avaliar a taxa de colonização em locais diferentes da pele do paciente com DA. Isto permitiu observar, em um mesmo paciente, a frequência de detecção de S.aureus e documentar que não houve diferença significante entre as lesões agudas, crônicas e vestíbulo nasal, havendo inclusive concordância entre o perfil de bactérias isoladas.

Estes resultados não são concordantes com alguns relatos prévios da literatura. Em 2006, GONG et al, mostraram que a cultura de áreas de pele lesionada apresentou maior densidade de S.aureus do que culturas de áreas de pele sã ${ }^{40}$. A avalição da densidade de S.aureus nas lesões, não foi contemplada no presente estudo, podendo ser um fator a justificar a 
ausência de diferenciação entre pele acometida e demais locais pesquisados. Em 2011, Yeung et al, detectaram que a frequência de S.aureus nas lesões úmidas foi maior do quem em lesões secas. Uma das justificativas para a ausência de diferenças entre os diversos locais é a possibilidade de contaminação, pois, apesar de serem lesões diferentes, são de um mesmo paciente e pelo prurido intenso e frequente acabam disseminando o S.aureus por todo o corpo.

Neste cenário, o presente estudo pode sugerir que, quando se quiser documentar a possibilidade de colonização, a coleta em apenas um local pode ser suficiente e os resultados estarão provavelmente mais relacionados à colonização que a uma infecção no local da lesão.

Um grande diferencial deste estudo foi a análise concomitante do swab nasal do paciente com DA e de seu cuidador primário, visto que até o presente momento não houve nenhum trabalho que fizesse este tipo de observação simultânea. A maior relevância, portanto foi demonstrar que os pacientes com dermatite atópica moderada ou grave e o seu cuidador primário concordam quanto ao perfil de colonização do S.aureus. Apesar da escassez de trabalhos relacionando colonização em pacientes com dermatite atópica associados à cuidadores primários, os estudos de Stephanie Fritz e colaboradores avaliaram alguns aspectos do ambiente dos pacientes colonizados. FRITZ et al em 2014 realizaram avaliação do ambiente de 50 crianças com cultura positiva para S.aureus, através da coleta de swab de superfícies ambientais de intenso compartilhamento tais como: roupa de cama, interruptores de luz e controles remotos. Foi 
demonstrado que $40 \%$ das amostras avaliadas apresentavam concordância dos achados do ambiente e dos pacientes, principalmente roupa de cama e controle remoto ${ }^{22}$. Assim como UHLEMANN et al, 2011, relatam $33 \%$ de concordância entre pacientes colonizados por S.aureus e cultura positiva em seu ambiente domiciliar ${ }^{32}$. Portanto estes dados nos apontam para um ambiente "adoecido" com a elevada colonização por S.aureus, atuando como um reservatório importante para a exposição a infecções e consequente exacerbações frequentes da DA. Ratificando que condutas terapêuticas devem ir além do olhar para o paciente com dermatite atópica, como também para o ambiente no qual o paciente esta inserido.

Apesar da importante colonização por S.aureus no ambiente domiciliar, o presente estudo não apresentou diferença estatisticamente significante entre o hábito do compartilhamento de produtos pessoais, toalhas e cama com a maior frequência de S.aureus. Entretanto, foi possível observar que há um elevado índice de compartilhamento de objetos pessoais e que orientações específicas neste sentido devem ser promovidas.

Em pacientes ambulatoriais como a maioria daqueles que apresentam dermatite atópica, espera-se uma maior prevalência de S.aureus MSSA. No presente estudo, a maior parte das cepas avaliadas foi meticilino sensível em todos os locais analisados (60\% swab nasal/ $66 \%$ swab lesão aguda/ 70\% swab lesão crônica). Estes resultados foram semelhantes ao estudo realizado por CAVALCANTE, no Brasil em 2015, que descreveu 60\% dos pacientes com DA colonizados por S.aureus MSSA no swab nasal ${ }^{21}$. Dados 
semelhantes foram encontrados por ONG et al,2010 e SCHNEIDER e colaboradores, 2013 que demonstraram um aumento na prevalência de portadores nasais de ate $80 \%$ entre as crianças ao redor do mundo ${ }^{7,9}$.

Apesar da elevada colonização por cepas do tipo MSSA, as cepas do tipo MRSA tem apresentado prevalência que variam de 0,5 a $18 \%$ nestes pacientes $^{20,36}$. CAVALCANTE et al, 2015 foi pioneiro no Brasil na identificação de S.aureus do tipo MRSA em 26,6\% dos pacientes com dermatite atópica. O alto nível encontrado pode estar relacionado com o perfil destes pacientes que frequentam regularmente um hospital de atendimento de doenças de elevada complexidade com alto índice de internações.

Quando avaliaram-se os cuidadores primários (indivíduos sem dermatite atópica) obteve-se $68 \%$ colonizados com S.aureus do tipo MSSA e $31,5 \%$ do tipo MRSA. Portanto um percentual de colonização mais elevado do que relatado da literatura em indivíduos saudáveis. UHLEMANN et al, 2011 em um estudo caso controle avaliaram fatores de risco sóciodemográficos e os reservatórios infecciosos associados às infecções por MRSA em membros de agregados familiares de indivíduos infectados por MRSA. E demonstraram colonização em swab nasal dos familiares de 56\% por MRSA em comparação no grupo controle apenas 38\% estavam colonizados $^{32}$. Dado semelhante foi relatado por Fritz et al, 2012, descrevendo que contatos domiciliares de crianças com infecções por S.aureus do tipo MRSA apresentaram maior prevalência de colonização, comparado com a população geral ${ }^{37}$. Esta elevada prevalência pode ser 
explicada por estudo realizado por SCOTT et al, 2008 , no qual afirmaram que o ambiente familiar é um importante meio para a contaminação e perpetuação do S.aureus. Neste estudo, foram avaliadas 35 residências de profissionais de saúde e 32 superfícies de suas residências. Amostras contaminadas com S.aureus foram isoladas em $97 \%$ dos domicílios, sendo $26 \%$ S.aureus do tipo MRSA ${ }^{38}$. Dados estes que reforçam a evidência do potencial de transmissão de infecções através de superfícies inanimadas e consequente maior colonização dos cuidadores primários. Contribuindo desta forma para a maior prevalência da colonização destes cuidadores como evidenciado neste estudo.

A gravidade da dermatite atópica avaliada pelo SCORAD não foi um fator de risco relevante em relação à colonização. O que concorda com Yeung et al, 2011, que avaliou 119 swab nasais e de pele de crianças com DA no Canadá, e não encontraram qualquer associação entre determinadas cepas de S.aureus e o aumento da gravidade da doença. Contudo estudo realizado na China por Kan-Lun et al, 2008, associa a colonização de 5 crianças por S.aureus MRSA a elevados índices de SCORAD, relacionado tal fato a presença do gene da leucociclina de Panton Valentine (PVL) presente no MRSA ${ }^{19}$. Porém, estudo randomizado realizado por Gong et al, 2006 demonstra a redução nos níveis do EASI (Eczema Área and Severity Índex) em crianças colonizadas com S.aureus, em acompanhamento ${ }^{40}$. A amostra reduzida de pacientes com dermatite atópica grave pode ser um dos fatores relacionados à ausência de correlação entre gravidade e colonização. 
A avaliação isolada do prurido nos pacientes com DA do presente estudo mostrou correlação significante com o percentual de colonização por cepas MRSA. Sabe-se que o prurido é o principal sintoma da DA e que afeta significativamente a qualidade de vida dos pacientes ${ }^{5}$. A infecção por S.aureus demonstra induzir rapidamente a expressão de IL 31 (que tem sido implicada no desenvolvimento de dermatite crônica em camundongos ${ }^{98}$ ), sugerindo que a colonização crônica e a superinfecção por este microrganismo podem contribuir para a piora do prurido ${ }^{97}$. Contudo, por não correlacionar quais patógenos estão mais associados a maiores scores de prurido, se faz necessário novas pesquisas para melhor entendimento desta associação.

Com relação ao perfil de resistência bacteriana em todos os locais analisados tanto no paciente como no cuidador primário as cepas se mostraram $100 \%$ sensíveis a antibióticos como linezolida, sulfametoxazol + trimetropin, rifampicina e vancomicin. A maior resistência foi para os antibióticos, penicilina (94\% de resistência), eritromicina (55\%) e clindamicina (25\%), o que coincide com estudo realizado por Gomes et al, 2010, onde demonstraram que $90 \%$ de S.aureus foram resistentes à penicilina, $53 \%$ resistentes a eritromicina e $47 \%$ a clindamicina. A boa surpresa foi a sensibilidade a sulfametoxazol + trimetropin, uma alternativa para tratamento domiciliar

Muitos dos aspectos observados neste estudo podem ser uteis para o entendimento da correlação entre S.aureus e dermatite atópica. A elevada prevalência de colonização e a preocupação com as cepas resistentes 
ratifica a necessidade de estratégias de combate a esta bactéria na pele dos pacientes com DA. Entretanto, estas estratégias devem contemplar mais medidas gerais de restauração da barreira cutânea, uso de produtos antissépticos e especialmente cuidados no domicilio que evitem grandes contaminações.

O reduzido número da amostra foi um fator limitante a avaliação de aspectos como gravidade da doença e colonização, mas ainda assim os resultados são relevantes para o entendimento desta importante correlação especialmente nos casos de dermatite atópica moderadas ou graves em seguimento em um hospital de alta complexidade. Estes resultados podem se juntar a outros estudos realizados no Brasil e auxiliar a compor um perfil de colonização do S.aureus em pacientes com dermatite atópica neste país. 
1. Houve uma elevada taxa de colonização por $S$ aureus em pacientes com dermatite atópica moderada ou grave em todos os locais pesquisados e. em mais de um terço da amostra, havia estafilococos meticilino resistentes

2. Os cuidadores primários com dermatite atópica apresentaram perfil de colonização de $S$ aureus coincidente com os pacientes associado a um grande compartilhamento de objetos pessoais

3. Não foi observada uma correlação entre a frequência de colonização e a gravidade da doença, embora índices de prurido elevados estivessem relacionados a maiores taxas de estafilococos meticilino resistentes 


\section{ANEXO I}

HOSPITAL DAS CLÍNICAS DA
FACULDADE DE MEDICINA DA Q pratil
USP - HCFMUSP

\section{PARECER CONSUBSTANCIADO DO CEP}

\section{DADOS DO PROJETO DE PESQUISA}

Título da Pesquisa: PERFIL DE COLONIZAÇĀO DE S.aureus EM PACIENTES COM DERMATITE ATÓPICA NAS LESÓES AGUDAS E CRÓNICAS

Pesquisador: ana paula beltran moschione castro

Área Temática:

Versăo: 1

CAAE: 57420816.0 .0000 .0068

Instituiçăo Proponente: HOSPITAL DAS CLINICAS DA FACULDADE DE MEDICINA DA U S P

Patrocinador Principal: Financiamento Proprio

DADOS DO PARECER

Número do Parecer: 1.637 .841

\section{Apresentaçăo do Projeto:}

Estudo de avallaçăo de flora mucosa de S.aureus em pacientes portadores de dermatite atopica.

Objetivo da Pesquisa:

Avallar o percentual de colonização dos pacientes com dermatite atopica na tase aguda e cronica da doença e, Identificar o perfil de resistencla das cepas de $S$ aureus presentes na pele destes pacientes $e$, correlacionar o pertll de colonizaçáo com a gravidade do quadro clinico,estado socioeconomico e habitos de higiene. Avaliar ainda se há diterenças no perfil de colonização nasal entre individuos do estudo com famillares que compartilham o mesmo domicillo

\section{Avaliaçăo dos Riscos e Beneficios:}

Os riscos são minimos, uma vez que a coleta de swab nasal é procedimento simples.

Comentários e Consideraçoes sobre a Pesquisa:

Não apresenta questionamentos de natureza etica.

Consideraçoes sobre os Termos de apresentaçăo obrigatória:

Aprovado com recomendação.

\section{Recomendaçoes:}

Especificar no projeto qual a falxa etaria que sera incluida na pesquisa.

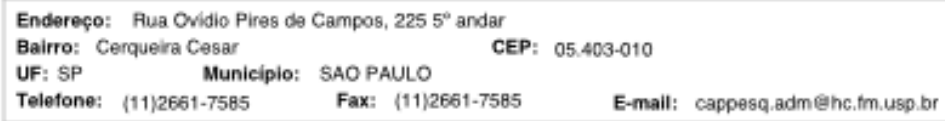




\title{
ANEXO II
}

\author{
Instituto Central \\ Hospital das Clínicas da \\ Faculdade de Medicina da Universidade de São Paulo \\ Av. Dr. Enéas de Carvalho Aguiar, 255 \\ CEP 05403-900 - São Paulo - Brasil
}

CEP - 023/2016

São Paulo, 27 de Janeiro de 2016

\section{DECLARAÇÃO DE ANUÊNCIA}

Informamos que do ponto de vista operacional é factível a realização dos exames laboratoriais do projeto intitulado "PERFIL DE COLONIZAÇÃO DE S.Aureus EM PACIENTES COM DERMATITE ATÓPICA NAS LESÕES AGUDAS E CRÔNICAS" tendo como Pesquisadora Principal Dra. Vanessa Tavares Pereira, Segunda Pesquisadora Dra. Maria Fernanda Badue Pereira e Orientadora Dra. Ana Paula Beltran Moschione Castro, lotadas no Departamento de Alergia e Imunologia do Instituto da Criança - HCFMUSP.

* Swab de fossas nasais anteriores e orofaringe

$\downarrow$ As cepas de S.Aureus isoladas serão congeladas para posterior análise molecular

+ Total de Pacientes: 30 pacientes e 30 coabitantes

$\downarrow$ Periodicidade das determinações: uma única dosagem por paciente que poder ser feita ao longo de seis meses.

- Duração total do projeto: 02 anos

DE ACORDO

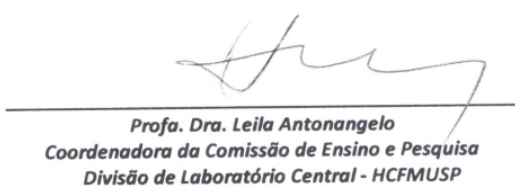




\title{
ANEXO III
}

\section{CritérioDiagnóstico de Hanifin e Rajka}

\author{
A-Critérios Clínicos Maiores ou Absolutos(3 ou mais): \\ $\square$ Prurido \\ $\square$ Morfologia e distribuição típica das lesões (envolvimento extensor e facial nas crianças e liquenificação e \\ linearidade flexural nos adultos) \\ $\square$ Dermatite crônica e recidivante \\ $\square$ História pessoal ou familiar de atopia \\ B-Critérios Clínicos Menores ou relativos \\ Exame Dermatológico \\ História Clínica: \\ Dados Complementares \\ Asteatose \\ $\square$ Hiperlinearidade palmar \\ Queratose pilar \\ Sinal de Hertogue (rarefação das \\ sombrancelhas) \\ $\square$ Prega infraorbital de Dennie- \\ Morgan \\ $\square$ Ptiríase Alba \\ Dermografismo branco \\ $\square$ Palidez ou eritema facial \\ $\square$ Quielite \\ Eczema de mamilo \\ $\square$ Pregas anteriores no pescoço \\ Acentuação perifolicular \\ $\square$ Escurecimento periorbital \\ $\square$ Alopecia areata \\ $\square$ Ictiose vulgar

\section{$\square$ Início precoce da doença $\quad \square$ Elevação da IgE sérica} \\ $\square$ Tendência a infecções $\square$ Hiperssensibilidade cutânea do tipo1 \\ cutâneas \\ $\square$ Conjuntivites recorrentes $\quad \square$ catarata \\ $\square$ Tendência a dermatites $\square$ ceratocone \\ inespecíficas de mãos e pés \\ $\square$ Curso influenciado por \\ fatores ambientais \\ $\square$ Curso influenciado por \\ fatores emocionais \\ $\square$ Hipersensibilidade alimentar \\ $\square$ Prurido com sudorese \\ $\square$ Urticária colinérgica \\ $\square$ Enxaqueca \\ $\square$ Hiperssensibilidade ao \\ níquel

Hanifin JM, Rajka G. Dianostic features of atopic dermatitis.ActaDerm Venereal Suppl (Stockh)1980;59:s44-7 


\section{ANEXO IV}

\section{DERMATITE ATÓPICA \\ Grupo Alergia e ImunologialCr - HCFMUSP}

NOME:

PRONTUÁRIO:

Sexo M F DN:___________ Idade: ___ Telefone para contato:

Mãe:

Idade:

Pai:

Idade

Endereço:

\section{História Clínica}

Início dos sintomas:

Periodicidade das crises(meses/ano):

Duração das crises:

Internação / infecção Sim Não

Freq

Caracterização do Quadro Clínico (Hanifin e Rajka):

Tempo de Aleitamento Materno: Sim Não Tempo: $<6$ meses $\geq 6$ meses

- Antecedente de Atopia

\begin{tabular}{|l|l|l|}
\hline Pessoais & $\begin{array}{l}\text { Asma Grave - Rinite Grave - Urticária Grave - Alergia Alimentar Grave } \\
\text { Outros: }\end{array}$ \\
\hline Familiares & $\begin{array}{l}\text { Pai : } \\
\text { Mãe: }\end{array}$ & Irmãos (descrever) : \\
\hline
\end{tabular}

\section{Tratamentos Prévios:}

- HIDRATANTE

Usa diariamente? Sim Não Em que horários?

Qual hidratante?

- CORTICOTERAPIA TÓPICA

Utilizou no último ano? Sim Não Duração do tratamento: Bandagem? Sim Não Tipo de corticosteróides tópicos utilizados

- CORTICOTERAPIA SISTEMICA:

Utilizou no último ano? Sim Não Duração do tratamento:

- ANTI-HISTAMÍNICOS

Utilizou Anti-histamínicos no último ano? Sim Não Houve boa resposta? Sim Não Duração do tratamento: contínuo Sim Não OUTROS MEDICAMENTOS: Imunossupressor Sim Não Outros 


\section{Outras Queixas:}

\section{EXAME FÍSICO}

Gravidade SCORAD: Leve (0-15) Moderada (15-50) Grave (>50)

\begin{tabular}{|l|l|}
\hline Critério & Intensidade \\
\hline 1.Eritema & \\
\hline 2.Edema/ Pápulas & \\
\hline 3.Crostas & \\
\hline 4.Escoriações & \\
\hline 5.Liquenificação & \\
\hline $\begin{array}{l}\text { 6.Ressecamento áreas sem } \\
\text { lesão }\end{array}$ & \\
\hline TOTAL & \\
\hline
\end{tabular}

A. Extensão:

B. Intensidade:

C. Sintomas subjetivos (prurido + perda de sono)

Total: $\mathrm{A} / 5+7 \mathrm{~B} / 2+\mathrm{C}=$

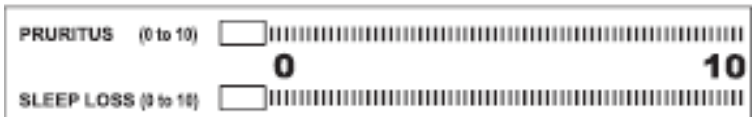

\section{Características das lesões da DA}
A. AGUDA:
B. Cronica:
C. Sinais de infecção aguda

\section{Perfil Socioeconomico}

Hábitos de higiene:

Quantos banho toma por dia ?

Quantas vezes na semana limpa a casa?

Quantas vezes limpa os banheiros?

Usa o lençol de cama por quantos dias?

Compartilha escova de dentes ?: Sim Não

Compartilha toalhas ?: Sim Não

Compartilha cama ?: Sim Não 
Compartilha produtos pessoais ( escova de cabelo, cortador de unha, utensílios de cozinha) ?: Sim Não

\section{Lava as mãos antes das refeições: Sim Não}

\section{Exame Físico:}

Localização e distribuição (vide SCORAD)

Infecção fúngica associada: Sim Não. Local

Infecção bacteriana associada? Sim Não. Local

Outros sinais relevantes do exame físico

Peso:

Z score:

Estatura: z escore: 
1. Huang JT, Abrams M, Tlougan B, Rademaker A, Paller AS. Treatment of Staphylococcus aureus Colonization in Atopic Dermatitis Decreases. Pediatrics. 2009;123(5):e808-14.

2. Solé D, Wandalsen GF, Camelo-Nunes IC, Naspitz CK; ISAAC Brazilian Group. Prevalence of symptoms of asthma, rhinitis, and atopic eczema among Brazilian children and adolescents identified by the International Study of Asthma and Allergies in Childhood (ISAAC)Phase 3. J Pediatr (Rio J). 2006;82(5):341-6.

3. Gesualdo F, Bongiorno D, Rizzo C, Bella A, Menichella D, Stefani S, Tozzi AE. MRSA Nasal Colonization in Children: Prevalence Metaanalysis, Review of Risk Factors and Molecular Genetics. Pediatr Infect Dis J. 2013;32(5):479-85.

4. Kim JP, Chao LX, Simpson EL, Silverberg JI. Persistence of atopic dermatitis (AD): A systematic review and meta-analysis. J Am Acad Dermatol. $2016 ; 75(4): 681-7$.

5. Manzoni AP, Pereira RL, Townsend RZ, Weber MB, Nagatomi AR, Cestari TF. Assessment of the quality of life of pediatric patients with the major chronic childhood skin diseases. An Bras Dermatol. 2012;87(3):361-8.

6. Amaral CS, March Mde F, Sant'Anna CC. Quality of life in children and teenagers with atopic dermatitis. An Bras Dermatol. 2012;87(5):71723.

7. Schneider L, Tilles S, Lio P, Boguniewicz M, Beck L, LeBovidge J, Novak N, Bernstein D, Blessing-Moore J, Khan D, Lang D, Nicklas R, Oppenheimer J, Portnoy J, Randolph C, Schuller D, Spector S, Tilles 
S, Wallace D. Atopic dermatitis: apractice parameter update 2012. J Allergy Clin Immunol. 2013;131:295-9.e1-27.

8. Wolter S, Price HN. Atopic dermatitis. Pediatr Clin North Am. 2014;61:241-60.

9. Ong PY, Leung DY. The infectious aspects of atopic dermatitis. Immunol Allergy Clin North Am. 2010;30(3):309-21.

10.Lopes C, Duarte AF, Correia O, Delgado L. Atopic dermatitis, innate immunity, and infection. Dermatol Online J. 2011;17(8):4.

11. Castro APM, Solé D, Rosário Filho NA, Jacob CM.. Practical guide for management of atopic dermatitis - conjunct opinion of allergologists from the Associação Brasileira de Alergia e Imunopatologia and Sociedade Brasileira de Pediatria. Rev Bras Alergia Imunopatol. 2006;29(6):268-82 .

12.Lipnharski C, d'Azevedo PA, Quinto VP, Bessa G, Bonamigo RR. Colonization by $\mathrm{S}$. aureus increases the EASI and the number of appointments by patients with atopic dermatitis: cohort with 93 patients. An Bras Dermatol. 2013;88(4):518-21.

13.Petry V, Lipnharski C, Bessa GR, Silveira VB, Weber MB, Bonamigo RR, d'Azevedo PA. Prevalence of community-acquired methicillinresistant Staphylococcus aureus and antibiotic resistance in patients with atopic dermatitis in Porto Alegre, Brazil. Int $J$ Dermatol. 2014;53(6):731-5.

14.Park HY, Kim CR, Huh IS, Jung MY, Seo EY, Park JH, Lee DY, Yang JM. Staphylococcus aureus Colonization in Acute and Chronic Skin Lesions of Patients with Atopic Dermatitis. Ann Dermatol. 2013;25(4):410-6. 
15. Alzolibani AA, Al Robaee AA, AI Shobaili HA, Bilal JA, Issa Ahmad M, Bin Saif G. Documentation of vancomycin-resistant Staphylococcus aureus (VRSA) among children with atopic dermatitis in the Qassim region, Saudi Arabia. Acta Dermatovenerol Alp Pannonica Adriat. $2012 ; 21(3): 51-3$.

16. Luger TA, Lotti T. Neuropeptides: role in inflammatory skin diseases. $J$ Eur Acad Dermatol Venereol. 1998;10(3):207-11.

17.Tang TS, Bieber T, Williams HC. Are the concepts of induction of remission and treatment of subclinical inflammation in atopic dermatitis clinically useful? J Allergy Clin Immunol. 2014;133(6):161525.

18. Keenan AL, Joan AG, Rachel MM. The role of S.aureus virulence factors in skin infection and their potential as vaccine antigens. Pathogens. 2016;5:22.

19. Hon KL, Leung AK, Kong AY, Leung TF, Ip M. Atopic dermatitis complicated by methicillin-resistant Staphylococcus aureus infection. $J$ Natl Med Assoc. 2008;100(7):797-800.

20.Farajzadeh S, rahnama ZZ, Kamyabi Z, Ghavidel B. bacterial colonization and Antibiotic resistance in children with atopic dermatitis. Dermatol On Line j. 2008;14:21.

21. Cavalcante FS, Abad ED, Lyra YC, Saintive SB, Ribeiro M, Ferreira DC, Santos KR. High prevalence of methicillin resistance and PVL genes among Staphylococcus aureus isolates from the nares and skin lesions of pediatric patients with atopic dermatitis. Braz $\mathrm{J}$ Med Biol Res. 2015;48(7):588-94. 
22. Fritz SA, Hogan PG, Singh LN, Thompson RM, Wallace MA, Whitney K, Al-Zubeidi D, Burnham CA, Fraser VJ. Contamination of environmental surfaces with Staphylococcus aureus in households with children infected with methicillin-resistant $S$ aureus. JAMA Pediatr. 2014;168(11):1030-8.

23. Hanifin JM, Rajka G. Diagnostic features of atopic dermatitis. Acta Derm Venereal Suppl (Stockh) 1980;59:s44-7

24.Severity Scoring of atopic dermatitis: the SCORAD index. Consensus Report of the European Task Force on Atopic Dermatitis. Dermatology. 1993;186:23-31.

25.Silverberg NB. A practical overview of pediatric atopic dermatitis, part 1: epidemiology and pathogenesis. Cutis. 2016;97(4):267-71.

26. Koubek et al. Whole-cell MALDI-TOF: Rapid screening method in environmental microbiology. International Biodeterioration\& Biodegradation, Prague 2012;69:82-6.

27. Goulart VAM, Resende RR. Maldi-tof: uma ferramenta revolucionária para as análises clínicas e pesquisa do câncer. Nonocell News. 2013;1(3).

28.Jorgensen JH, Ferraro MJ. Antimicrobial Susceptibility Testing: A Review of General Principles and Contemporary Practices.2009.

29. de Benedictis FM, Franceschini F, Hill D, Naspitz C, Simons FE, Wahn U, Warner JO, de Longueville M; EPAAC Study Group.. The allergic sensitization in infants with atopic eczema from different countries. Allergy. 2009;64(2):295-303. 
30. Gao L, Grant AV, Rafaels N, Stockton-Porter M, Watkins T, Gao P, Chi P, Muñoz M, Watson H, Dunston G, Togias A, Hansel N, Sevransky J, Maloney JP, Moss M, Shanholtz C, Brower R, Garcia JG, Grigoryev DN, Cheadle C, Beaty TH, Mathias RA, Barnes KC. Polymorphisms in the myosin light chain kinase gene that confer risk of severe sepsis are associated with a lower risk of asthma. J Allergy Clin Immunol. 2007;119(5):1111-8.

31.Palmer CN, Irvine AD, Terron-Kwiatkowski A, Zhao Y, Liao H, Lee SP, Goudie DR, Sandilands A, Campbell LE, Smith FJ, O'Regan GM, Watson RM, Cecil JE, Bale SJ, Compton JG, DiGiovanna JJ, Fleckman $P$, Lewis-Jones S, Arseculeratne G, Sergeant A, Munro CS, El Houate B, McElreavey K, Halkjaer LB, Bisgaard H, Mukhopadhyay $\mathrm{S}$, McLean WH. Common loss-of-function variants of the epidermal barrier protein filaggrin are a major predisposing factor for atopic dermatitis. Nat Genet. 2006;38(4):441-6.

32. Uhlemann AC, Knox J, Miller M, Hafer C, Vasquez G, Ryan M, Vavagiakis $P$, Shi $Q$, Lowy FD. The environment as an unrecognized reservoir for community-associated methicillin resistant Staphylococcus aureus USA300: a case-control study. PLoS One. $2011 ; 6(7): \mathrm{e} 22407$.

33. Boguniewicz M, Leung DY. Recent insights into atopic dermatitis and implications for management of infectious complications. J Allergy Clin Immunol. 2010;125(1):4-13.

34. Kisich KO, Carspecken CW, Fieve S, Boguniewicz M, Leung DY. Defective killing of Staphylococcus aureus in atopic dermatitis is associated with reduced mobilization of human beta-defensin-3. $J$ Allergy Clin Immunol. 2008;122:62-8. 
35. De Benedetto A, Agnihothri R, McGirt LY, Bankova LG, Beck LA. Atopic dermatitis: a disease caused by innate immune defects? J Invest Dermatol. 2009;129:14-30.

36. Suh L, Coffin S, Leckerman KH, Gelfand JM, Honig PJ, Yan AC. Methicillin-resistant Staphylococcus aureus coloniza- tion in children with atopic dermatitis. Pediatr Dermatol. 2008;25:528-34.

37. Fritz SA, Hogan PG, Singh LN, Thompson RM, Wallace MA, Whitney K, Al-Zubeidi D, Burnham CA, Fraser VJ. Contamination of environmental surfaces with Staphylococcus aureus in households with children infected with methicillin-resistant $S$ aureus. JAMA Pediatr. 2014;168(11):1030-8.

38. Scott et al (2008)

39. Yeung M, Balma-Mena A, Shear N, Simor A, Pope E, Walsh S, McGavin MJ. Identification of major clonal complexes and toxin producing strains among Staphylococcus aureus associated with atopic dermatitis. Microbes Infect. 2011;13(2):189-97.

40.Gong JQ, Lin L, Lin T, Hao F, Zeng FQ, Bi ZG, Yi D, Zhao B. Skin colonization by Staphylococcus aureus in patients with eczema and atopic dermatitis and relevant combined topical therapy: a doubleblind multicentre randomized controlled trial. $\mathrm{Br} J$ Dermatol. 2006;155(4):680-7.

41.Dillon SR, Sprecher C, Hammond A, Bilsborough J, RosenfeldFranklin M, Presnell SR, Haugen HS, Maurer M, Harder B, Johnston J, Bort S, Mudri S, Kuijper JL, Bukowski T, Shea P, Dong DL, Dasovich M, Grant FJ, Lockwood L, Levin SD, LeCiel C, Waggie K, 
Day H, Topouzis S, Kramer J, Kuestner R, Chen Z, Foster D, ParrishNovak J, Gross JA. Interleukin 31, a cytokine produced by activated $T$ cells, induces dermatitis in mice. Nat Immunol. 2004;5(7):752-60.

42. Sonkoly E, Muller A, Lauerma Al, Pivarcsi A, Soto H, Kemeny L, Alenius $\mathrm{H}$, Dieu-Nosjean MC, Meller $\mathrm{S}$, Rieker J, Steinhoff $M$, Hoffmann TK, Ruzicka T, Zlotnik A, Homey B. IL-31: a new link between T cells and pruritus in atopic skin inflammation. $J$ Allergy Clin Immunol. 2006117(2):411-7.

43. Gomes PL, Malavige GN, Fernando N, Mahendra MH, Kamaladasa SD, Seneviratne JK, Karunatilaka DH, Ogg GS. Characteristics of Staphylococcus aureus colonization in patients with atopic dermatitis in Sri Lanka. Clin Exp Dermatol. 2011;36(2):195-200. 